\title{
A Weight Restricted DEA Model for the Supplier Evaluation and Selection
}

\author{
Xiao-guang Qi \& Bo Guo \\ College of Information System and Management, National University of Defense Technology, Changsha, \\ 410073, China
}

\begin{abstract}
Supplier evaluation and selection is an important issue in the supply chain management. Many different factors would have effect on the performance of suppliers, therefore supplier evaluation and selection is a typical multi-criteria decision making problem. Data Envelopment Analysis (DEA) is an effective methodology of evaluating the relative efficiency of Decision Making Units (DMUs) with multiple inputs and outputs. DEA has been widely used in the supplier evaluation and selection problem. However, traditional literatures mostly concentrated on the supplier evaluation and selection without consideration of the decision maker's preference. In this paper, we propose a weight restricted DEA model for the supplier evaluation and selection problem in which two kinds of decision maker's preferences can be reflected by introducing different kinds of weight restrictions. Numerical example is provided to examine the validity and effectiveness of our proposed model.
\end{abstract}

KEYWORD: data envelopment analysis; supplier evaluation; supplier selection; weight restriction

\section{INTRODUCTION}

Choosing a good supplier would have great benefit on the performance of the whole supply chain and supplier evaluation and selection has always been a valuable topic in the supply chain management [1]. Data Envelopment Analysis (DEA) was first introduced by Charnes et al. [2] in 1978 and DEA is an effective methodology of evaluating the relative efficiency of Decision Making Units (DMUs) with multiple inputs and outputs. It is obvious that the supplier evaluation and selection problem can be formulated as a multi-criteria decision making problem [1], and therefore DEA can be used for solving this problem and a great literatures can be found in this field.

DEA has been widely used in the supplier evaluation and selection problem. Ho et al. [1] proposed a literature review on the multi-criteria approaches for the evaluation and selection of suppliers in which DEA is included. Chai et al. [3] proposed another review on these approaches and pointed out that DEA is one of the most popular methods. Liu et al. [4] combined DEA with AHP as an integrated method for the supplier selection in supply chain management. A structured methodology was proposed by Chen [5] in which DEA and some other methods were combined together. Dotoli et al. [6] proposed a hierarchical extension of the DEA for optimal supplier selection in multiple sourcing contexts. Besides, Abdelfattah et al. [7] introduced a theoretical framework for the dyadic supply chains efficiency evaluation.

However, the traditional literatures mostly concentrated on the supplier evaluation and selection without consideration of the Decision Maker's preference [8]. Zhao et al. [8] proposed a preference restraint DEA approach for supplier selection in which the decision maker's preference on inputs and outputs are reflected by the AHP method. Weight restrictions can also be used for the decision maker's preference. Saen [9] proposed a DEA based decision model in which ordinal data, weight restrictions and non-discretionary factors were considered simultaneously. Saen [10] also proposed another weight restriction DEA model for the supplier selection decisions with the consideration of dual-role factors. Pablo et al. [11] introduced a new multi-criteria approach for the analysis of efficiency by modeling decision maker preferences.

In this paper, we propose a weight restricted DEA model for the supplier evaluation and selection problem in which the Decision Maker's preference is considered and reflected by introducing the weight restrictions. The rest of this paper is organized as follow: in section 2, we give some preliminary about the weight restricted DEA; in section 3, our proposed model is introduced in which two kinds of 
decision maker's preferences have been considered; in section 4, a numerical example is proposed for the examination of our proposed model; finally, in section 5 , we give the conclusions.

\section{PRELIMINARY}

In the DEA methodology, it is supposed that there are $n$ DMUs (such as the suppliers) with $m$ inputs and $s$ outputs, the vectors $x_{j}=\left[x_{1 j}, x_{2 j}, \ldots, x_{m j}\right]^{\mathrm{T}}$ and $y_{j}=\left[y_{1 j}, y_{2 j}, \ldots, y_{s j}\right]^{\mathrm{T}}$ are used to denote the inputs and outputs of $D M U_{j}$, in which $j=$ $1,2, \ldots, n$. The basic efficiency model for $D M U_{j_{0}}\left(j_{0}=1,2, \ldots, n\right)$ is named as CCR model as follows:

$\operatorname{Max} h_{j_{0}}=\sum_{r=1}^{s} u_{r} y_{r j_{0}}$

s.t.

$\sum_{r=1}^{s} u_{r} y_{r j}-\sum_{t=1}^{m} v_{t} x_{t j} \leq 0, j=1,2, \ldots, n$

$\sum_{t=1}^{m} v_{t} x_{t j_{0}}=1$

$u_{r} \geq 0, r=1,2, \ldots, s$

$v_{t} \geq 0, t=1,2, \ldots, m$

in which $u=\left[u_{1}, u_{2}, \ldots, u_{s}\right]^{\mathrm{T}} \quad$ and $v=\left[v_{1}, v_{2}, \ldots, v_{m}\right]^{\mathrm{T}}$ are the optimal weights assigned to the outputs and inputs respectively, and $h_{j_{0}}$ is the efficiency score of $D M U_{j_{0}}$.

In the traditional DEA model, the DMU is allowed to get its most favorable optimal weights and therefore many inputs and outputs would be assigned with zero weights. These zero weights would be somewhat unrealistic in practice and weight restrictions were introduced to reduce these zero weights.

The first weight restricted DEA model was introduced by Dyson et al. [12] as follows:

$\left\{\begin{array}{l}u_{r} \geq \varepsilon, r=1,2, \ldots, s \\ v_{t} \geq \varepsilon, t=1,2, \ldots, m\end{array}\right.$

in which $\varepsilon$ is the allowable minimum weight.

Assurance region model [13] is another kind of weight restricted DEA model which is able to reflect the decision maker's preference information on inputs and outputs. The weight restriction in assurance region model is in the form of ratios between different weights and it can be transformed into the linear form as follows:

$\left\{\begin{array}{l}u_{r_{0}}-\varepsilon u_{r_{1}} \geq 0, r_{0}, r_{1}=1,2, \ldots, s \\ v_{t_{0}}-\varepsilon v_{t_{1}} \geq 0, t_{0}, t_{1}=1,2, \ldots, m\end{array}\right.$
Based on the concept of virtual weights, which means the combination of the multiplier weight and the output (input) data, Wong et al. [14] proposed the virtual weight restriction model as follows:

$$
\left\{\begin{array}{l}
u_{r_{0}} y_{r_{0} j_{0}}-\varepsilon \sum_{r=1}^{s} u_{r} y_{r_{j_{0}}} \geq 0, r_{0}=1,2, \ldots, s \\
v_{t_{0}} x_{t_{0} j_{0}}-\varepsilon \sum_{t=1}^{m} v_{t} x_{t_{j_{0}}} \geq 0, t_{0}=1,2, \ldots, m
\end{array}\right.
$$

\section{PROPOSED MODEL FOR THE SUPPLIER EVALUATION AND SELECTION}

In this section, a weight restricted DEA model is proposed for the supplier evaluation and selection problem in which the decision maker's preference has been considered and reflected by the weight restrictions. As far as we can say, there are two different kinds of decision maker's preference: the first kind is the preference on a certain input or output; and the second kind is the preference on a certain DMU. In our proposed model, these two kinds of decision maker's preferences can be considered simultaneously by introducing some weight restrictions.

\subsection{Preference on a certain input or output}

Weight restrictions can be used to reflect the decision maker's preference on inputs and outputs [13]. In this paper, we combined the assurance region method [13] and the virtual weights method [14] together to reflect the decision maker's preference on inputs and outputs respectively.

It is supposed that, for all DMUs, the decision maker prefer a certain input $x_{t_{0}}\left(t_{0}=1,2, \ldots, m\right)$ to the other inputs. Then the following virtual weight restrictions will be introduced to reflect this preference:

$v_{t_{0}} x_{t_{0} j}-\varepsilon_{t}\left(v_{t} x_{t j}\right) \geq 0, t=1,2, \ldots, m, j=1,2, \ldots, n$

in which $\varepsilon_{t}$ is the measure of how much decision maker prefers input $x_{t_{0}}$ to the other inputs. Similarly, if decision maker prefers a certain output $y_{r_{0}}\left(r_{0}=1,2, \ldots, s\right)$ to the other outputs, then the following weight restrictions will be used:

$u_{r_{0}} y_{r_{0} j}-\tau_{r}\left(u_{r} y_{r j}\right) \geq 0, r=1,2, \ldots, s, j=1,2, \ldots, n$

in which $\tau_{r}$ is the measure of how much decision maker prefers output $y_{r_{0}}$ to the other outputs.

\subsection{Preference on a certain DMU}

The traditional weight restriction models mostly concentrated on the research of decision maker's 
preference on inputs and outputs [13], while pay little attention on the research of decision maker's preference on a certain DMU. In this section, we propose a weight restricted DEA model based on the super efficiency model for reflecting the decision maker's preference on a certain DMU.

Our proposed model is based on the super efficiency model which was first introduced by Andersen et al. [15]. The super efficiency of $D M U_{j_{0}}\left(j_{0}=1,2, \ldots, n\right)$ is calculated by excluding it from the DMUs set as follows [15]:

$\operatorname{Max} \theta_{j_{0}}=\sum_{r=1}^{s} u_{r j_{0}} y_{r_{j_{0}}}$

s.t.

$\sum_{r=1}^{s} u_{r} y_{r j}-\sum_{t=1}^{m} v_{t} x_{t j} \leq 0, j=1,2, \ldots, n, j \neq j_{0}$

$\sum_{t=1}^{m} v_{t} x_{t j_{0}}=1$

$u_{r} \geq 0, r=1,2, \ldots, s$

$u_{r j_{0}} \geq 0, r=1,2, \ldots, s$

$v_{t} \geq 0, t=1,2, \ldots, m$

in which $\theta_{j_{0}}$ is the super efficiency of $D M U_{j_{0}}$. By the application of super efficiency model, there would be a set of super efficiency scores $\left\{\theta_{1}, \theta_{2}, \ldots, \theta_{n}\right\}$. Then we use $\theta_{\max }$ to denote the maximum super efficiency which means that $\theta_{\max }=\max \left\{\theta_{1}, \theta_{2}, \ldots, \theta_{n}\right\}$.

A DMU with smaller inputs and bigger outputs would be evaluated as a better DMU, and therefore the decision maker's preference on a certain DMU can be reflected by the weight restriction on inputs or on outputs respectively. In our proposed model, the decision maker's preference on a certain $D M U_{j_{0}}\left(j_{0}=1,2, \ldots, n\right)$ is reflected by the weight restriction on outputs as follows:

$u_{r j_{0}}-w_{j_{0}} u_{r}=0,1 \leq w_{j_{0}} \leq \frac{\theta_{\max }}{\theta_{j_{0}}}$

in which $w_{j_{0}}$ means the decision maker's preference on $D M U_{j_{0}}$ to the other DMUs. It should be noted that the bigger this value is, the more $D M U_{j_{0}}$ is preferred to the other DMUs by the decision maker.

\subsection{An integrated model for the supplier evaluation and selection}

In this section, we propose an integrated model for the supplier evaluation and selection problem in which both two kinds of decision maker's preferences could be reflected.

Without loss of generality, it is supposed that the decision maker prefers input $x_{t_{0}}$ to the other inputs and prefers $D M U_{j_{0}}$ to the other DMUs. Then the efficiency evaluation model for $D M U_{j_{0}}$ would be as follows:

$\operatorname{Max} \theta_{j_{0}}=\sum_{r=1}^{s} u_{r j_{0}} y_{r j_{0}}$

s.t.

$\sum_{r=1}^{s} u_{r} y_{r j}-\sum_{t=1}^{m} v_{t} x_{t j} \leq 0, j=1, \ldots, n, j \neq j_{0}$

$\sum_{t=1}^{m} v_{t} x_{t j_{0}}=1$

$u_{r j_{0}}-w_{j_{0}} u_{r}=0, r=1, \ldots, s$

$v_{t_{0}} x_{t_{0} j}-\varepsilon_{t}\left(v_{t} x_{t j}\right) \geq 0, t=1, \ldots, m, j=1, \ldots, n$

$u_{r} \geq 0, r=1, \ldots, s$

$u_{r j_{0}} \geq 0, r=1, \ldots, s$

$v_{t} \geq 0, t=1, \ldots, m$

Similarly, the efficiency model for $D M U_{j_{1}}\left(j_{1} \neq\right.$ $j_{0}$ ) with the preference on $x_{t_{0}}$ and $D M U_{j_{0}}$ would be as follows:

$\operatorname{Max} \theta_{j_{1}}=\sum_{r=1}^{s} u_{r} y_{r j_{1}}$

s.t.

$\sum_{r=1}^{s} u_{r} y_{r j}-\sum_{t=1}^{m} v_{t} x_{t j} \leq 0, j=1, \ldots, n, j \neq j_{0}, j_{1}$

$\sum_{r=1}^{s} u_{r j_{0}} y_{r j_{0}}-\sum_{t=1}^{m} v_{t} x_{t j_{0}} \leq 0$

$\sum_{t=1}^{m} v_{t} x_{t j_{1}}=1$

$u_{r j_{0}}-w_{j_{0}} u_{r}=0, r=1, \ldots, s$

$v_{t_{0}} x_{t_{0} j}-\varepsilon\left(v_{t} x_{t j}\right) \geq 0, t=1, \ldots, m, j=1, \ldots, n$

$u_{r} \geq 0, r=1, \ldots, s$

$u_{r j_{0}} \geq 0, r=1, \ldots, s$

$v_{t} \geq 0, t=1, \ldots, m$

\section{NUMERICAL EXAMPLE}

In this section, a numerical example is provided to examine the validity and effectiveness of our proposed model. This example is selected from [10] in which ten suppliers are considered as the DMUs with two inputs and two outputs as follows:

Input 1: (TC) Total Cost of shipment;

Input 2: (NS) Number of Shipments per month;

Output 1: (NOT) Number of shipments to arrive On Time;

Output 2: (NB) Number of Bills received without error. 
The inputs and outputs data and the super efficiency without preference are provided in Table 1 as the benchmark of our proposed model. And it is supposed that the decision maker prefers input NS to input TC and prefers DMU2 to the other DMUs, then the efficiency evaluation result by our proposed model is provided in Table 2, in which we set $w_{j_{0}}=1.0324$ and $\varepsilon_{t}=100$.

Table 1. Inputs and outputs of ten suppliers

\begin{tabular}{|c|c|c|c|c|c|}
\hline \multirow{2}{*}{ DMU } & \multicolumn{2}{|c|}{ Inputs } & \multicolumn{2}{c|}{ Outputs } & \multirow{2}{*}{$\begin{array}{c}\text { Super } \\
\text { efficiency }\end{array}$} \\
\cline { 2 - 5 } & TC & NS & NOT & NB & 0.9747 \\
\hline 1 & 253 & 197 & 187 & 90 & 1.0116 \\
\hline 2 & 268 & 198 & 194 & 130 & 1.0776 \\
\hline 3 & 259 & 229 & 220 & 200 & 1.0465 \\
\hline 4 & 180 & 169 & 160 & 100 & 0.9955 \\
\hline 5 & 257 & 212 & 204 & 173 & 0.9829 \\
\hline 6 & 281 & 173 & 165 & 163 & 0.0279 \\
\hline 7 & 291 & 193 & 188 & 185 & 1.027 \\
\hline 8 & 334 & 177 & 168 & 85 & 0.9687 \\
\hline 9 & 249 & 185 & 177 & 130 & 0.9794 \\
\hline 10 & 216 & 176 & 167 & 160 & 1.0156 \\
\hline
\end{tabular}

Table 2. Efficiency evaluation by our proposed model

\begin{tabular}{|c|c|c|c|c|}
\hline \multirow{2}{*}{ DMU } & \multicolumn{2}{|c|}{ Model (7) } & \multicolumn{2}{c|}{ Our proposed model } \\
\cline { 2 - 5 } & Efficiency & Ranking & Efficiency & Ranking \\
\hline 1 & 0.9747 & 9 & 0.9388 & 8 \\
\hline 2 & 1.0116 & 5 & 1.0729 & 1 \\
\hline 3 & 1.0776 & 1 & 0.9793 & 4 \\
\hline 4 & 1.0465 & 2 & 0.9374 & 10 \\
\hline 5 & 0.9955 & 6 & 0.9732 & 5 \\
\hline 6 & 0.9829 & 7 & 0.9829 & 3 \\
\hline 7 & 1.0279 & 3 & 1.0212 & 2 \\
\hline 8 & 0.9687 & 10 & 0.9383 & 9 \\
\hline 9 & 0.9794 & 8 & 0.9535 & 7 \\
\hline 10 & 1.0156 & 4 & 0.9725 & 6 \\
\hline
\end{tabular}

As it is shown in Table 2, the ranking of suppliers has changed by introducing the decision maker's preference. On the one hand, as the decision maker prefers DMU2 to the other DMUs, then the ranking of DMU2 has been promoted from the 5th to the 1st. On the other hand, the decision maker prefers input NS to input TC and therefore the ranking of DMU1 and DMU9 has also risen because their good performance on the input NS. We can say that the validity and effectiveness of our proposed model has been verified by the application into this numerical example.

\section{CONCLUSIONS}

The supplier evaluation and selection is an important issue in the supply chain management which has great effect on the performance of the whole supply chain. DEA has been widely used as an efficiency evaluation method in the supplier evaluation and selection problem, however most literatures neglect the influence of decision maker's preference. In this paper, we proposed a weight restricted DEA model for the supplier evaluation and selection problem in which the decision maker's preference can be reflected by introducing different weight restrictions. As far as we can say, there are mainly two kinds of preferences: the first kind is the preference on a certain input or output; and the second one is the preference on a certain DMU. We use an integrated model to reflect these two kinds of references simultaneously. The validity and effectiveness of our proposed model has been verified by the application into a real data example.

\section{REFERENCES}

[1] W. Ho, X. Xi, P. K. Dey. Multi-criteria decision making approaches for supplier evaluation and selection: A literature review. European Journal of Operational Research, vol. 202, 16-24, 2010.

[2] A. Charnes, W. W. Cooper, E. Rhodes. Measuring the efficiency of decision making units. European Journal of Operational Research, vol. 2, 429-444, 1978.

[3] J. Chai, J. Liu, E. Ngai. Application of decision-making techniques in supplier selection: A systematic review of literature. Expert Systems with Applications, vol. 40, 3872-3885, 2013.

[4] J. Liu, C. Wu. An integrated method for supplier selection in SCM. Proceedings of ICSSSM, 617-620 2005.

[5] Y. Chen. Structured methodology for supplier selection and evaluation in a supply chain. Information Sciences, vol. 181, 1651-1670, 2011.

[6] M. Dotoli, M. Falagario. A hierarchical model for optimal supplier selection in multiple sourcing contexts. International Journal of Production Research, vol. 50, 2953-2967, 2012.

[7] W. Abdelfattah, A. Rebai. Dyadic supply chains efficiency: A new theoretical framework to consider members power relationship and decision makers preferences. ICMSAO, 2013.

[8] X. Zhao, L. Sun. A preference restraint DEA approach for supplier selection. WiCOM, 2008.

[9] R. F. Saen. A decision model for ranking suppliers in the presence of cardinal and ordinal data, weight restrictions, and nondiscretionary factors. Annual of Operation Research, vol. 172, 177-192, 2009.

[10] R. F. Saen. Restricting weights in supplier selection decisions in the presence of dual-role factors. Applied Mathematical Modelling, vol. 34, 2820-2830, 2010.

[11] D. Pablo, R. Palomares, A. M. Prieto. A new multicriteria approach for the analysis of efficiency in the Spanish olive oil sector by modeling decision maker preferences. European Journal of Operational Research, vol. 234, 241-252, 2014.

[12] Dyson R. G., E. Thanassoulis. Reducing weight flexibility in data envelopment analysis. Journal of the Operational Research Society, vol. 39(6), 563-576, 1988.

[13] Thompson R. G., L. N. Langemeier, C. Lee, E. Lee, R. M. Thrall. The role of multiplier bounds in efficiency analysis with application to Kansas farming. Journal of Econometrics, vol. 46, 93-108, 1990.

[14] Wong Y. H. B., J. E. Beasley. Restricting weight flexibility in data envelopment analysis. Journal of Operational Research Society, vol. 41(9), 829-835, 1990.

[15] P. Andersen, N. C. Petersen. A procedure for ranking efficient units in data envelopment analysis. Management Science, vol. 39, 1261-1264, 1993. 